\title{
MIMO-OFDM Channel Estimation in Rayleigh and Rician Fading based on MMSE Estimator
}

\author{
Maisnam Babita Devi ${ }^{1}$, Kaustubh Bhattacharyya ${ }^{2}$ \\ Dept of Electronics and Communication Engineering, Assam Don Bosco University, Azara, Guwahati, Assam, India ${ }^{1,2}$
}

\begin{abstract}
OFDM is the combination of both multiplexing and modulation. In this paper, orthogonal frequency division multiplexing (OFDM) is considered for wideband transmission to mitigate intersymbol interference and enhance system capacity. The main principle of an OFDM is the insertion of Guard Intervals over a multipath fading channel, where two ways are possible; one method being Zero Padding and another being addition of Cyclic Prefix. This paper is mainly focused on the significance of cyclic prefix. Also, the combination of OFDM with MIMO is one of the most promising candidates for future communication systems, ranging from wireless LAN to broadband access, which is presently under study. This paper starts with comparisons of OFDM using QPSK and QAM on different channels, followed by modeling the MMSE estimators through a $4 \times 4$ MIMO system on MATLAB. Inthe end, results of different simulations are compared to conclude that MMSE algorithm provides comparatively better results with the implementation of MIMO-OFDM system.
\end{abstract}

Keywords: OFDM, Cyclic prefix, ISI, Orthogonality, BER, MIMO.

\section{INTRODUCTION}

To provide high data rates at better Quality of Service (QoS) in mobile environments is one of the main challenges of the fourth generation wireless system [1]. Also, it has been made necessary to develop the methods to increase spectral efficiency and the search for innovative solutions, as within the fast shrinking spectrum available for wireless communication, multipath components have been added destructively [2]. MIMO-OFDM is a combination of multiplexing and antenna diversity. Combining the two schemes provide superior data rate, range and reliability without requiring additional bandwidth or transmit power.

OFDM can be said as a technique which has high data rate transmission potential with efficiency of high bandwidth and has ability to fight against multipath fading [3]. OFDM uses non-overlapping adjacent channel to increase spectral efficiency and allows multiple carriers be used to transmit different symbols with spectral overlap while ensuring coexistence of nearby signals due to orthogonality. Most importantly, OFDM can efficiently deal with ISI problems.

MIMO (Multiple Input Multiple Output) system can be said to be a very useful technique which offer superior data rates, range and reliability without requiring additional bandwidth or transmit power.By using several antennas at both the transmitter and receiver, MIMO systems create multiple independent channels for sending multiple data streams. Different versions of MIMO systems are available viz. SISO (Single Input Single Output), SIMO(Ingle Input Multiple Output) and MISO(Multiple Input Single Output).

This paper aims to compare the performance of Rayleigh and Rician fading channels using QPSK and QAM modulations in MIMO environment where channel estimation is considered. Channel estimation becomes necessary as the number of parallel wireless links increases with the increase of antennas. This paper describes MMSE (Minimum Mean Square Error) estimators.In Section II, the basic system model of OFDM, MIMO and the equalizer are discussed. In section III, experimental results are presented. Concluding remarks are given in section IV.

\section{II.MIMO-OFDM SYSTEM}

(a) OFDM system

OFDM is a combination of modulation and multiplexing. OFDM is a special case of frequency division multiplexing (FDM). As an analogy, a FDM channel is like water flow out of a faucet, in contrast the OFDM is like a shower. In a faucet all water comes in one big stream and cannot be sub-divided. OFDM shower is made up of a lot of little streams. Now, if I put my thumb over the faucet hole, I can stop the water flow but I cannot do the same for the shower. So although both do the same thing, they respond differently to interference. OFDM technology can effectively reduce the ISI [4]. 


\section{IJIREEICE

OFDM is a method of digital modulation in which a signal is split into several narrowband channels at different frequencies. Conceptually, OFDM is a specialized FDM, the additional constraint being the entire carrier signals are orthogonal to each other. In OFDM, the sub-carrier frequencies are chosen so that the sub-carriers are orthogonal to each other meaning that cross-talk between the sub-channels is eliminated and inter carrier guard bands are not required. The required bandwidth is efficiently reduced by compressing the multiple modulated carriers together by OFDM, while keeping the modulated signals orthogonal, due to which they do not interfere with each other [5]. This greatly simplifies the design of both the transmitter and the receiver, unlike conventional FDM; a separate filter for each sub-channel is not required. As shown in Figure 1, it does not use individual band limited filters and oscillators for each sub channel and furthermore, the spectra of subcarriers are overlapped for bandwidth efficiency.

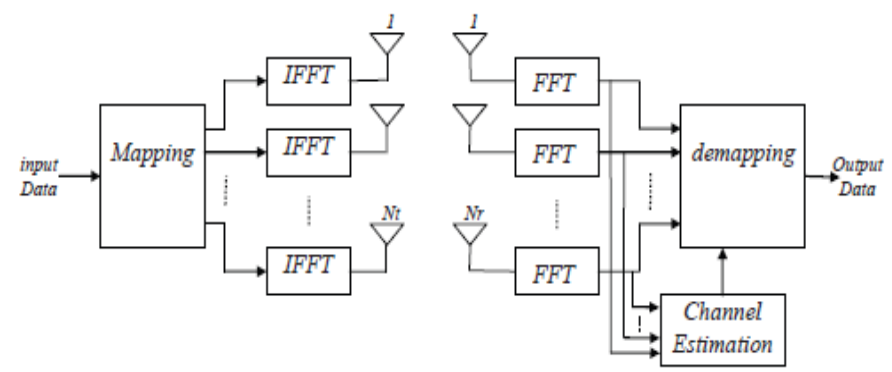

Fig-1.OFDM transmission scheme implemented using IFFT/FFT

The binary information is being generated from uniformly distributed random integers with equal probability of either 0 or 1 given as [3]:

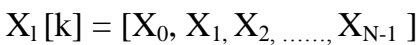

Where, $1=0,1,2, \ldots ., \infty$

and $\mathrm{k}=0, \ldots \ldots, \mathrm{N}-1$

The use of orthogonal subcarriers allows the spectra of the subcarriers to overlap which increases the spectral efficiency. Despite their overlapping spectrum, it is still possible to recover the individual subcarrier's signals, if the orthogonality is maintained [6]. If the time-limited complex exponential signals are considered to be $\left\{\mathrm{e}^{\mathrm{j} 2 \mathrm{f}}{ }_{\mathrm{k}}\right\}^{\mathrm{N}-1}{ }_{\mathrm{k}=0}$ representing the different subcarriers at $\mathrm{f}_{\mathrm{k}}=\mathrm{k} / \mathrm{T}_{\text {sym }}$ in the OFDM signal, where $0 \leq \mathrm{t} \leq \mathrm{T}_{\text {sym }}$, then these signals are defined to be orthogonal if the integral of the products for their common (fundamental) period is zero [7], that is,

$$
\frac{1}{\mathrm{~T}_{\text {sym }}} \int_{0}^{\mathrm{T}_{\text {sym }}} \mathrm{e}^{\mathrm{j} 2 \pi \mathrm{f}_{\mathrm{k}} \mathrm{t}} \mathrm{e}^{-\mathrm{j} 2 \pi \mathrm{f}_{\mathrm{i}} \mathrm{t}} \mathrm{dt}=\frac{1}{\mathrm{~T}_{\text {sym }}} \int_{0}^{\mathrm{T}_{\text {sym }}} \mathrm{e}^{\mathrm{j} 2 \pi \frac{\mathrm{k}}{\mathrm{T}_{\text {sym }}} \mathrm{t}} \mathrm{e}^{-\mathrm{j} 2 \pi \frac{\mathrm{i}}{\mathrm{T}_{\text {sym }}} \mathrm{t}} \mathrm{dt}
$$

$=\frac{1}{\mathrm{~T}_{\text {sym }}} \int_{0}^{\mathrm{T}_{\text {sym }}} \mathrm{e}^{\mathrm{j} 2 \pi \frac{(\mathrm{k}-\mathrm{i})}{\mathrm{T}_{\text {sym }}} \mathrm{t}} \mathrm{dt}$

$=\left\{\begin{array}{rr}1, & \forall \text { integerk }=\mathrm{i} \\ 0, & \text { otherwise }\end{array}\right.$

The above orthogonality is an essential condition for the OFDM signal to be ISI-free.

OFDM transmitter maps the message bits into a sequence of PSK or QAM symbols which will be subsequently converted into $\mathrm{N}$ parallel streams. Each of $\mathrm{N}$ symbols from serial-to-parallel (S/P) conversion is carried out by the different subcarrier. Let $\Psi_{l . k}(t)$ denote the $\mathrm{l}_{\text {th }}$ OFDM signal at the kth subcarrier, which is given as

$\Psi_{1 . k}(t)=\left\{\begin{array}{cl}\left.\mathrm{e}^{\mathrm{j} 2 \pi \mathrm{f}_{\mathrm{k}}(\mathrm{t}-\mathrm{lT}} \mathrm{T}_{\text {sy } \mathrm{m}}\right), & 0 \leq \mathrm{t} \leq \mathrm{T}_{\text {sym }} \\ 0, & \text { otherwise }\end{array}\right.$

Then the pass band and baseband OFDM signals in the continuous-time domain can be expressed respectively as

$\mathrm{x}_{\mathrm{l}}(\mathrm{t})=\operatorname{Re}\left\{\frac{1}{\mathrm{~T}_{\text {sym }}} \sum_{\mathrm{l}=0}^{\infty}\left\{\sum_{\mathrm{k}=0}^{\mathrm{N}-1} \mathbf{x}_{\mathrm{l}}[\mathbf{k}] \Psi_{\mathrm{l} . \mathrm{k}}(\mathrm{t})\right\}\right\}$ 
and $x_{l}(\mathrm{t})=\sum_{l=0}^{\infty} \sum_{k=0}^{N-1} \boldsymbol{x}_{\boldsymbol{l}}[\boldsymbol{k}] e^{j 2 \pi f_{k}\left(t-l T_{s y m}\right)}$

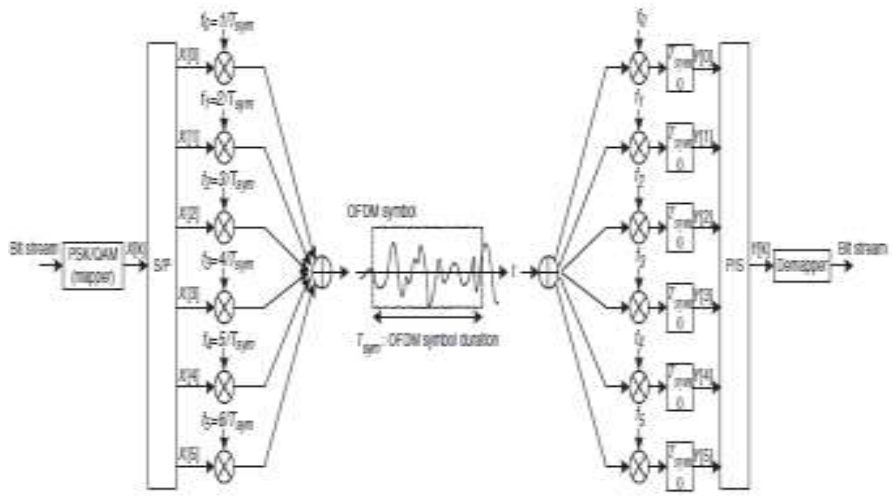

Fig-2. OFDM modulation/demodulation

IFFT is done to convert frequency domain data into the time domain signal [8]. Figure 2 shows a diagram of the OFDM modulation/demodulation, including the IFFT and FFT operations. The main principle behind an OFDM is the insertion of guard intervals where are two ways. One is the zero padding (ZP) and other is the cyclic extension. In this work, cyclic extension is done using cyclic prefix where the last symbol of the OFDM symbol is copied into its front.

(b)MIMO system

MIMO technique is used for providing high data rate, reliability and requires no additional bandwidth for transmission [9].MIMO takes the advantage of multipath as it uses multiple antennas to send multiple parallel signals (from transmitter). In the wireless spectrum, a major limitation is that, with the increased capacity, the spectrum and/ or transmitting power should also increase. To overcome this problem, the concept of using multiple antennas at both the transmitting and receiving ends was introduced. It has become a current theme of international wireless research. MIMO involves Space Time Transmit Diversity (STTD), Spatial Multiplexing (SM) and Uplink Collaborative MIMO. A general block diagram is shown below where multiple antennas are used in both the transmitter and receiver ends.

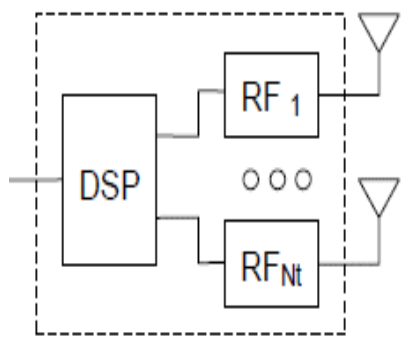

TX

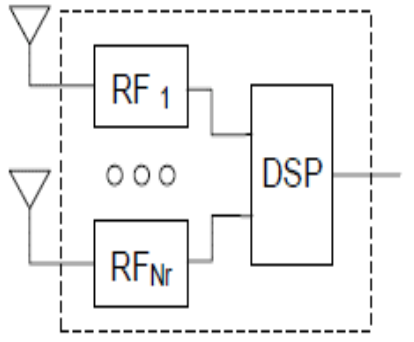

$\mathrm{RX}$

Fig- 3. MIMO wireless system

The MIMO system model can be expressed as

$$
y=H x+n
$$

where $y$ and $x$ are the receive and transmit vectors respectively. In addition, $H$ and $n$ are the channel matrix and the noise factor respectively. If $H_{i j}$ is the channel from $j^{\text {th }}$ transmit antenna TXj to $i^{\text {th }}$ receive antenna RXi and if we consider $\mathrm{i}=\mathrm{j}=4$, then the matrix representation would be:
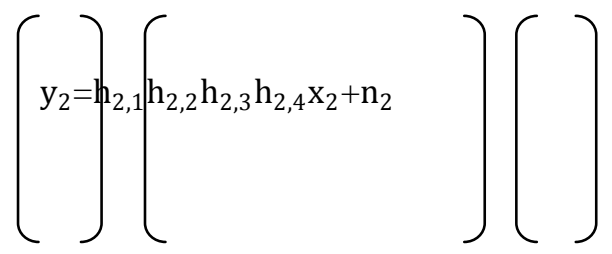

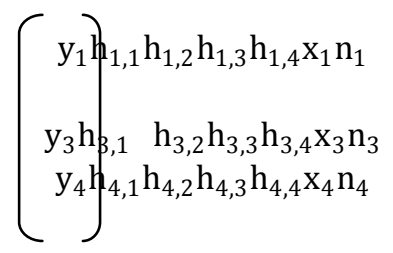




\section{International Journal of Innovative Research in} Electrical, Electronics, Instrumentation and Control Engineering

\section{ISO 3297:2007 Certified}

Vol. 5, Issue 5, May 2017

\section{EXPERIMENTAL RESULTS}

Considering the above discussion, addition of OFDM is done with both Rayleigh and Rician channels, using QPSK modulation and QAM modulation taking one after another.A Rayleigh environment assumes NLOS (no line-of-sight) whereas if there is a LOS (line-of-sight), then it can be modelled by Rician fading. Both have the same characteristics except for a zero expected value in case of Rayleigh fading and a non-zero expected value for a Rician fading [g].For the simulation of basic OFDM system, we used the following parameters as shown in Table-1.

\section{Table-1:}

\begin{tabular}{|l|c|}
\hline \multicolumn{1}{|c|}{ PARAMETERS } & SPECIFICATIONS \\
\hline FFT size $(\mathrm{n})$ & 64 \\
\hline Constellation & QPSK/QAM \\
\hline Channel Model & Rayleigh/ Rician \\
\hline No. of taps & $\mathrm{n} / 4$ \\
\hline Cyclic Prefix $\left(\mathrm{N}_{\mathrm{cp}}\right)$ & 52 \\
\hline No. of used subcarriers & 3 \\
\hline No. of symbols per frame & \\
\hline
\end{tabular}

The Fig-4(a) and Fig-4 (b) shows the comparison of BER with different SNR's on QPSK constellation using 2 different channel models i.e Rayleigh fading and Rician fading channels respectively. For small SNR values the calculated BER is quite large due to relative high power of noise.

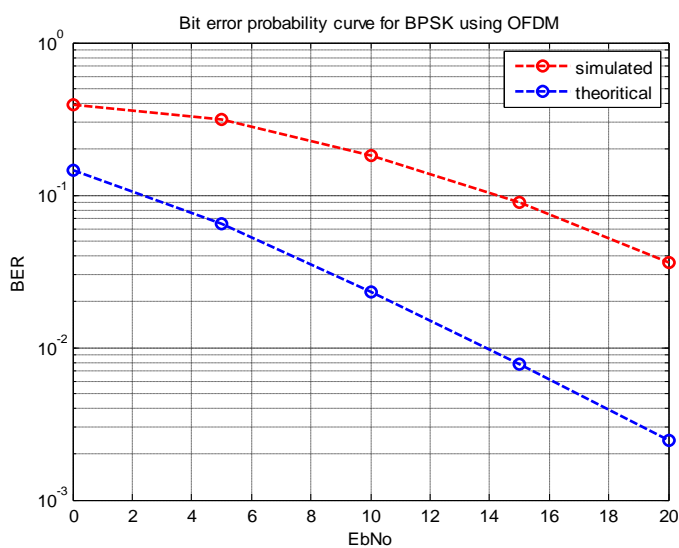

(a)

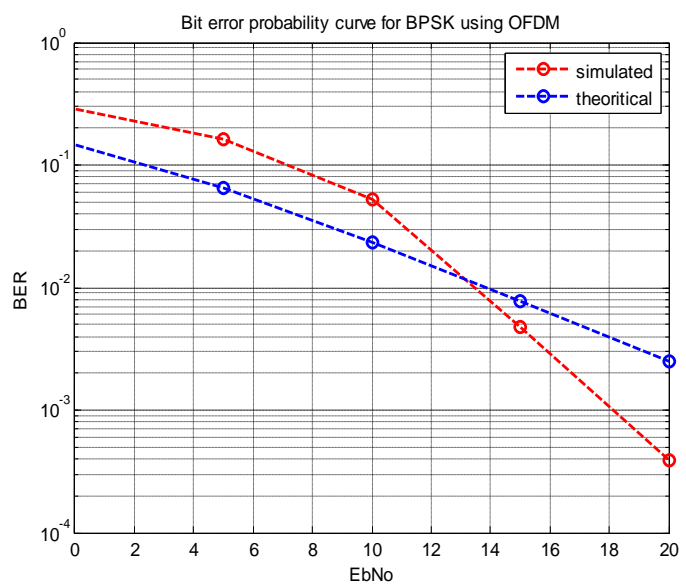

(b)

Fig-4. BER plot (a) QPSK in Rician fading with OFDM and MMSE and (b) QPSK in Rayleigh fading with OFDM and MMSE 


\section{International Journal of Innovative Research in} Electrical, Electronics, Instrumentation and Control Engineering

\section{ISO 3297:2007 Certified}

Vol. 5, Issue 5, May 2017

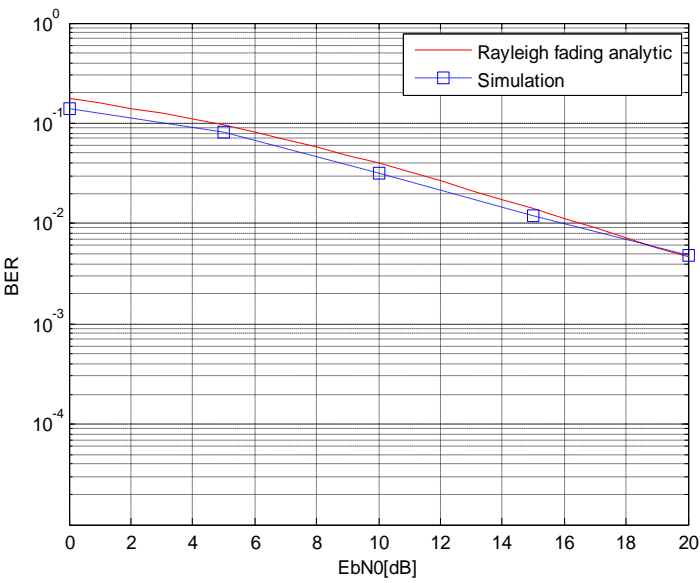

(a)

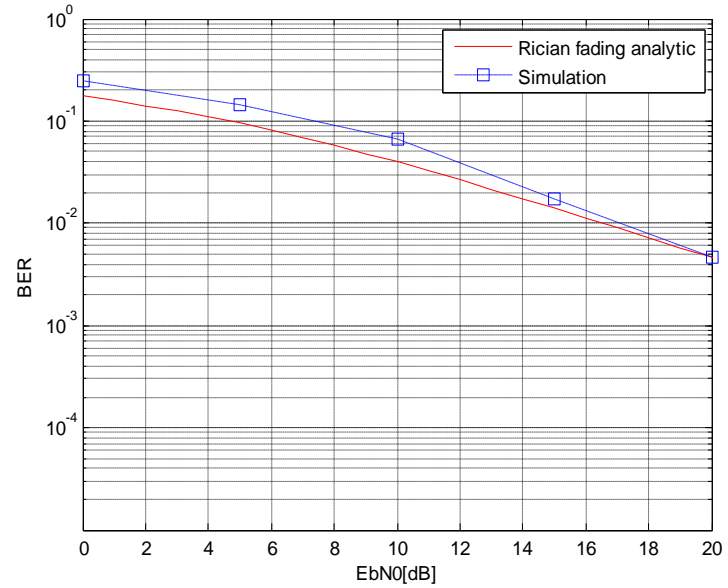

(b)

Fig-5. BER plot (a)QAM in Rayleigh fading with OFDM \& MMSE equalization and (b) QAM in Rician fading with OFDM \& MMSE equalization

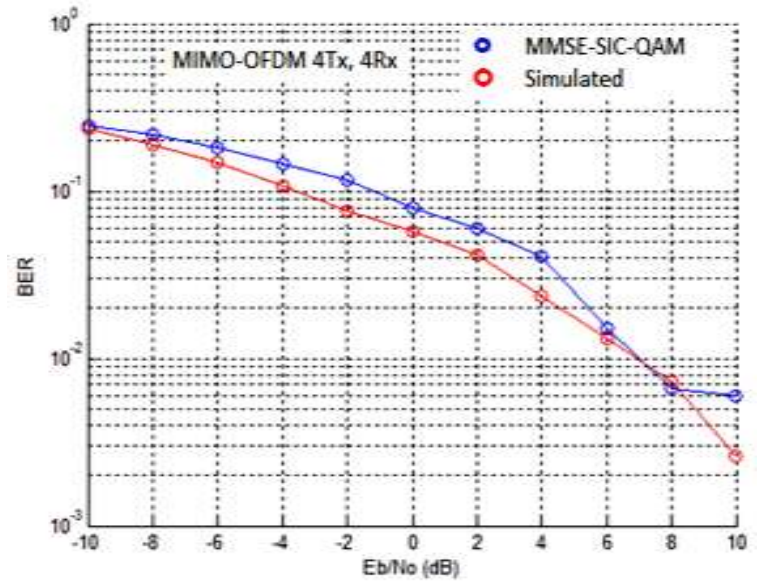

Fig-6. BER plot for QAM inRayleigh fading using OFDM-MIMO with MMSE

As SNR is increased the BER decreases as shown. And the Bit Error rate (BER) curves are based on the mean square errors of the channel estimation. For the calculation of BER, the simulation makes use of the formulae calculated earlier. So, while comparing the above two BER plots, it can be concluded that, as in Fig 4 (a) i.e Rician fading channel, for $\mathrm{Eb} / \mathrm{No}=10 \mathrm{db}$, the bit error rate is above $10^{-1}$ and in case of Rayleigh fading channel, Fig 4 (b), it is near to $10^{-1}$ which is better than Rician channel. Similarly for QAM, again the BER determines how many of the received bits are in error, and then computes it by the number of bits in error divided by the total no of bits in the transmitted signal. 


\section{IJIREEICE \\ International Journal of Innovative Research in \\ Electrical, Electronics, Instrumentation and Control Engineering \\ ISO 3297:2007 Certified \\ Vol. 5, Issue 5, May 2017}

\section{CONCLUSION}

Here we try to achieve maximum gain by sending copies of the same OFDM through a 4x4 MIMO System. This is also called transmit diversity in OFDM systems. Maximum Ratio Combining was then used to boost the received estimated signal. Different configurations of the parameters were investigated to examine the effect on the received BER values. The choice of modulation scheme depends on the scenario with respect to the factors of transmission required. For instance, QAM transmit more symbols than QPSK and BPSK. The 4x4 MIMO System was investigated with receiver architecture based on MMSE receiver. As expected, due to increasing the total number of transmitted symbols per second, there would be an increase in the total number of errors and therefore the need to operate at a higher SNR values. The combination of OFDM with MIMO shows a form of flooring in the curve which signifies the need for additional coding to fully utilise the OFDM scheme. This flooring is a result of both inter-subcarrier and inter-antenna interference.From the above plots it can be concluded that, the comparison made among the considered modulation schemes in this work, in Figure 4 and 5, under the considered fading channels, when OFDM is implemented, shows that the combination of QAM with Rayleigh gives better performance.

Therefore, again this combination is examined using MMSE equalizers, and from Figure 6, it can be said that, using MMSE schemewith MIMO-OFDM in Rayleigh with the QAM modulation schemes, performance is better as compared with the other configurations.

\section{REFERENCES}

[1] Ouarzazi et al, "Channel estimation of OFDM system for high data rate communications on mobile environments", No. 978 Vol. 1, pp-42445347, April 2009, IEEE.

[2] Kandarpa Kumar Sarma and Abhijit Mitra, "Estimation of MIMO Wireless Channels using Artificial Neural Networks", Department of Electronics and Communication Engineering Indian Institute of Technology (IIT) Guwahati.

[3] Sajjad Ahmed Ghauri, et al, "Implementation of OFDM and Channel Estimation using LS and MMSE Estimators", International Journal of Computer and Electronics Research, Volume 2, Issue 1, February 2013.

[4]Friss H.T. (1946) "A note on simple transmission formula". Proc. IRE, No. 34, Vol. 5, pp- 254-256.

[5] Neetu Sood and Ajay K Sharma, "BER Performance of OFDM-BPSK and -QPSK over Nakagami-m Fading Channels", No. 978, Vol. 1, pp4244-4791, June 2010, IEEE.

[6] Charan Langton, "Orthogonal Frequency Division Multiplexing Tutorial”. www.complextoreal.com [Retrived on 22-05-13].

[7] Yong Soo Cho, Jaekwon Kim, Won Young Yang, Chung-Gu Kang, “ MIMO-OFDM wireless communications with MATLAB”, John Willey \& Sons (Asia), Pte Ltd., 2010.

[8] Gupta et al, "Bit Error Rate Performance in OFDM System Using MMSE \& MLSE Equalizer Over Rayleigh Fading Channel Through The BPSK, QPSK,4 QAM \& 16 QAM Modulation Technique”, Vol. 1, Issue 3, pp.1005-1011, Journal of Engineering Research and Applications (IJERA).

[9] A. Omri and R. Bouallegue, "New Transmission Scheme For Mimo-Ofdm System", International Journal of Next-Generation Networks (IJNGN) Vol.3, No.1, March 2011. 